\title{
Persistent Fever and Pancytopenia: Lupus Flare vs Macrophage Activation Syndrome
}

Amy McGhee, MD

Thomas Jefferson University, amy.mcghee@jefferson.edu

Follow this and additional works at: https://jdc.jefferson.edu/tmf

Part of the Medicine and Health Sciences Commons

Let us know how access to this document benefits you

\section{Recommended Citation}

McGhee, MD, Amy (2015) "Persistent Fever and Pancytopenia: Lupus Flare vs Macrophage Activation Syndrome," The Medicine Forum: Vol. 16, Article 11.

DOI: https://doi.org/10.29046/TMF.016.1.010

Available at: https://jdc.jefferson.edu/tmf/vol16/iss1/11

This Article is brought to you for free and open access by the Jefferson Digital Commons. The Jefferson Digital Commons is a service of Thomas Jefferson University's Center for Teaching and Learning (CTL). The Commons is a showcase for Jefferson books and journals, peer-reviewed scholarly publications, unique historical collections from the University archives, and teaching tools. The Jefferson Digital Commons allows researchers and interested readers anywhere in the world to learn about and keep up to date with Jefferson scholarship. This article has been accepted for inclusion in The Medicine Forum by an authorized administrator of the Jefferson Digital Commons. For more information, please contact: JeffersonDigitalCommons@jefferson.edu. 


\section{Persistent Fever and Pancytopenia: Lupus Flare vs Macrophage Activation Syndrome}

Amy McGhee, MD

\section{INTRODUCTION}

Macrophage activation syndrome (MAS), first named in 1993, is a subcategory of hemophagocytic lymphohistiocytosis $(H L H)$, characterized by prolonged fever, hepatosplenomegaly, pancytopenia, liver dysfunction, and most notably hyperferritinemia. MAS, in particular, is the term used to describe secondary HLH, or HLH caused by rheumatologic conditions. Secondary HLH can also be associated with other systemic autoimmune diseases, underlying malignancy, infection, or medications and can be a life-threatening complication. MAS is characterized by unwarranted proliferation and stimulation of $T$ cells and benign macrophages leading to an excessive inflammatory state with hypersecretion of cytokines. This, in turn, leads to phagocytosis of normal blood cells and injury to the organs containing these macrophages (liver, spleen, bone marrow, lymph nodes). With regard to rheumatologic conditions, MAS is most often seen associated with juvenile idiopathic arthritis (incidence of $7-13 \%)$ while it is much less commonly seen in systemic lupus erythematosus (SLE) with an incidence of 0.9-4.6\%. The etiology is unknown, although there are hypotheses.

\section{CASE PRESENTATION}

A 53 year old female with SLE complicated by lupus nephritis and lupus anticoagulant initially presented with a syncopal episode. While walking out of the rheumatologist office, she felt faint and had an assisted fall to the floor with transient loss of consciousness. The patient reported approximately 1-2 months of decreased oral intake, weight loss, daily fevers to $102-103^{\circ} \mathrm{F}$, lightheadedness, dry cough, and 1 week of rash on her upper chest. During this time, her rheumatologist adjusted her SLE medications, attributing her symptoms to a SLE flare. Work up during this admission (labs listed in Table 1 ) ruled out sepsis as a source of fever. It was determined that her symptoms and lab abnormalities were likely secondary to a lupus flare given her fever, rash, constitutional symptoms, and pancytopenia in the setting of SLE that was difficult to control. Early macrophage activation syndrome (MAS) was also on the differential given the elevated ferritin level. Her symptoms improved with pulse dose steroids and resuming mycophenolate mofetil for immunosuppression. The plan was to change this to cyclosporine as an outpatient to prevent MAS.

Before switching to cyclosporine however, the patient was readmitted for recurrent fevers, fatigue, diarrhea, and worsening lab abnormalities. On admission, her physical exam was significant for fever to $102.4^{\circ} \mathrm{F}$, tachycardia, and dry mucus membranes. Labs are shown in Table 2. The most remarkable finding was a ferritin of 11,741 which was a significant increase from 4,542 during her last admission. The patient underwent a bone marrow biopsy, which demonstrated increased macrophages with hemophagocytic activity, confirming a diagnosis of MAS.

\section{DIFFERENTIAL DIAGNOSIS}

In an SLE patient with recent flare 4 months ago, a recurrent lupus flare was highest on the differential as the patient reported joint pain, skin rash, fevers, weight loss, and fatigue. Infection is also high on the differential for a patient on chronic immunosuppression who presents with fever. An infectious workup was undertaken including a Right upper quadrant ultrasound, chest CT. blood cultures, urine cultures, respiratory viral panel, and transthoracic echo, all of which were negative for infection. Anti-dsDNA antibodies were unexpectedly normal and complement levels were unremarkable which made a SLE flare less likely.

During the initial admission, the hyperferritinemia, an acute phase reactant, was thought to be elevated in the setting of a lupus flare or infection. However, the dramatic increase to 11,741 upon readmission raised suspicion for MAS. Multiple case reports have described this diagnostic difficulty in distinguishing between MAS and a SLE flare. It has been reported that elevation of ferritin (greater than or equal to 500ug/L) and LDH can help differentiate as this represents activation of the mononuclear phagocyte system. Hyperferritinemia has a sensitivity and specificity of almost 100\% for distinguishing between a SLE flare and MAS. Lastly, a bone marrow biopsy is usually diagnostic for MAS if there is still uncertainty. 
Table 1. Labs for first admission.

\begin{tabular}{|c|c|c|c|c|c|}
\hline \multirow{2}{*}{$\begin{array}{l}\text { Lab } \\
\text { WBC (B/L) }\end{array}$} & \multicolumn{2}{|c|}{ Result } & \multirow{2}{*}{$\begin{array}{l}\text { Lab } \\
\text { AST }(U / L)\end{array}$} & \multicolumn{2}{|c|}{ Result } \\
\hline & 1.2 & L & & 71 & $H$ \\
\hline Hemoglobin (g/dL) & 7.4 & $L$ & $\operatorname{ALT}(U / L)$ & 23 & \\
\hline Platelets (B/L) & 178 & & Alk phosphatase $(U / L)$ & 35 & \\
\hline Sodium (mmol/L) & 137 & & Lactate (mmol/L) & 1.1 & \\
\hline Potassium (mmol/L) & 4.4 & & Iron (mcg/dL) & 44 & \\
\hline Chloride (mmol/L) & 103 & & $\begin{array}{l}\text { Iron Binding Capacity (mcg/ } \\
\mathrm{dL} \text { ) }\end{array}$ & 179 & $L$ \\
\hline Bicarbonate (mmol/L) & 22 & $L$ & Iron Saturation (\%) & 25 & \\
\hline BUN (mg/dL) & 21 & & Ferritin (ng/mL) & 4542 & $H$ \\
\hline Creatinine (mg/dL) & 0.9 & & $\mathrm{ESR}(\mathrm{mm} / \mathrm{hr})$ & 114 & $\mathrm{H}$ \\
\hline Glucose (mg/dL) & 127 & & $\mathrm{CRP}(\mathrm{mg} / \mathrm{dL})$ & 1.50 & $H$ \\
\hline PTT (sec) & 42 & $\mathrm{H}$ & C3 complement (mg/dL) & 79 & $L$ \\
\hline $\mathrm{PT}(\mathrm{sec})$ & 10.1 & & C4 complement (mg/dL) & 12 & \\
\hline INR & 0.93 & & Anti-dsDNA Ab (IU) & 25 & \\
\hline Total Protein (g/dL) & 5.1 & $L$ & Haptoglobin (mg/dL) & 295 & $H$ \\
\hline Albumin (g/dL) & 2.8 & $L$ & $\mathrm{LDH}(I \cup / L)$ & 396 & $H$ \\
\hline Total bilirubin (mg/dL) & 0.2 & & Vitamin B12 (pg/mL) & 433 & \\
\hline \multirow[t]{4}{*}{ Direct bilirubin (mg/dL) } & $<0.2$ & & Folate $(n g / m L)$ & $>20$ & \\
\hline & & & Urinalysis & \multicolumn{2}{|c|}{$2+$ protein } \\
\hline & & & & \multicolumn{2}{|c|}{ Trace blood } \\
\hline & & & & \multicolumn{2}{|c|}{$1 \mathrm{RBC} 4 \mathrm{WBC}$} \\
\hline
\end{tabular}

Table 2. Labs upon second admission.

\begin{tabular}{|c|c|c|c|c|c|}
\hline Lab & \multicolumn{2}{|c|}{ Result } & Lab & \multicolumn{2}{|c|}{ Result } \\
\hline WBC (B/L) & 0.9 & $L$ & AST (U/L) & 128 & $H$ \\
\hline Hemoglobin (g/dL) & 8.5 & $L$ & $\mathrm{ALT}(\mathrm{U} / \mathrm{L})$ & 49 & $\mathrm{H}$ \\
\hline Platelets (B/L) & 72 & $L$ & Alk phosphatase (U/L) & 41 & \\
\hline Total Protein (g/dL) & 5.6 & $L$ & Ferritin (ng/mL) & 11741 & $H$ \\
\hline Albumin (g/dL) & 3.1 & L & LDH (IU/L) & 1135 & $H$ \\
\hline Total bilirubin (mg/dL) & 0.6 & & & & \\
\hline
\end{tabular}




\section{McGhee, MD: Persistent Fever and Pancytopenia}

\section{OUTCOME AND FOLLOW-UP}

Once the diagnosis of MAS was confirmed, the patient was treated with cyclosporine, anakinra (IL-1 receptor blocker), and high dose steroids. These were uptitrated until the ferritin, leukopenia, and liver function tests began to improve. Interestingly, in the work-up of the patient's pancytopenia, her EBV studies showed a positive VCA IgG and ENA IgG so the patient was also treated with IVIG in case an EBV infection was contributing to the MAS. The patient was discharged home after a month-long hospitalization. In the next nine months, her medications were all gradually tapered and she had a concordant improvement in ferritin, blood counts, and symptoms.

\section{DISCUSSION}

There remain many uncertainties surrounding MAS including its etiology, treatment, and prognosis. In one case study of a patient with SLE and MAS, the patient tested positive for numerous antibodies (ANA, anti-dsDNA, anti-Sm, anti-RNP, anti-Ro, and peripheral ANCA antibodies). It was hypothesized that the overwhelming amount of autoantibodies and immune complexes bound to normal blood cells trigger phagocytosis of those cells. It is possible that the quantity of these autoantibodies and the increased complement activity during a SLE flare can trigger MAS.

Once the diagnosis of MAS is made, it is important to initiate therapy quickly as it can be a life-threatening illness. The cornerstone of therapy is high dose steroids. Multiple case reports also discuss the importance of immunosuppressant medications, such as cyclosporine A and cyclophosphamide in patients who do not respond to steroids, such as the patient in this report. One study, in particular, demonstrated $54 \%$ of patients with MAS from systemic autoimmune disease who received only high dose steroids were resistant to this treatment and the majority of patients who were treated with both steroids and immunosuppression had good treatment response. Another case report demonstrated the fairly rapid improvement in labs and clinical condition of a young man who was treated with cyclosporine when he did not respond to steroids. IVIG is another treatment option which is especially useful in patients who are suspected to have an underlying viral infection. Further, the next line of treatment if both steroids and immunosuppression are not effective is biologic agents, such as anakinra and rituximab. Anakinra is an IL-1 receptor blocker that has been used effectively in patients with MAS secondary to SLE which did not respond to steroids, IVIG, and cyclosporine. There is also evidence that rituximab is effective in treating resistant MAS. The patient in this case study did not respond appropriately to pulse dose steroids, cyclosporine, or IVIG, so she was started on anakinra and did well after a few weeks on this regimen.

Further, prognosis is another area of uncertainty in MAS. There have been no prognostic indicators established yet but there is evidence suggesting the level of certain cytokines can be associated with a worse prognosis and that ferritin levels may help predict outcome. Multiple studies have found no correlation between ferritin levels and the severity of disease or outcome; however, serial ferritin levels may shed more light on prognosis. The patient in this case report had a ferritin of 4500 on initial admission, which increased to 11,000 on her subsequent admission indicating lack of response to therapy. Once anakinra was added and titrated, her symptoms improved and her ferritin slowly decreased over the course of weeks. She was safely discharged home with close follow up.

\section{KEY POINTS}

MAS is a potentially life-threatening complication of rheumatologic diseases, characterized by excessive activation of benign macrophages which lead to the phagocytosis of normal blood cells and injury to surrounding tissues. Common clinical manifestations include fever, pancytopenia, liver dysfunction, hepatosplenomegaly, and hyperferritinemia. Diagnosis can be difficult as the signs and symptoms of MAS can mimic an infection or SLE flare. However, hyperferritinemia and elevated LDH are characteristic of MAS and may help distinguish and guide treatment decisions. If uncertainty remains, a bone marrow biopsy can usually establish the diagnosis. The best treatment options at this point include high dose steroids, immunosuppression, IVIG, and biologics depending on the patient's laboratory and clinical response. 
The Medicine Forum, Vol. 16 [2015], Art. 11

\section{REFERENCES}

1. Atteritano M, David A, Bagnato G, Beninati C, Frisina A, laria C, Bagnato G, Cascio A. Haemophagycytic syndrome in rheumatic patients. A systematic review. European Review for Medical and Pharmacological Sciences. 2012; 16: 1414-1424

2. Bakshi J, Hassan S, D'Cruz D, Chan C. Rituximab therapy in refractory macrophage activation syndrome secondary to systemic lupus erythematosus. Lupus. 2013; 22: 1544-1546.

3. Dubuc CAE, Ecenarro MU, Villalba CM, Cåceres VA, Rubio $\|_{\text {, }}$ Otano JB. Hemophagocytic syndrome as the initial manifestation of systemic lupus erythematosus. Reumatologia Clinica. 2014; 10(5): $321-324$

4. Jung S. Hemophagocytic syndrome diagnosed by liver biopsy in a female patient with systemic lupus erythematosus. Journal of Clinical Rheumatology. 2013; 19(8): 449-451

5. Kim JM,Kwok SK, Ju JH, Park KS, Park GS, Kim HY, Park SH. Macrophage activation syndrome resistant to medical therapy in a patient with systemic lupus erythematosus and its remission with splenectomy. Rheumatol Int. 2013; 33: 767-771.

6. Maruyama J and Inokuma S. Cytokine profiles of macrophage activation syndrome associated with rheumatic diseases. The Journal of Rheumatology. 2010; 37(5): 967-973.

7. Vilaiyuk S, Sirachainan N, Wanitkun S, Pirojsakul K, Vaewpanich J. Recurrent macrophage activation syndrome as the primary manifestation in systemic lupus erythematosus and the benefit of serial ferritin measurements: a case-based review. Clinical Rheumatology. 2013; 32: 899-904.

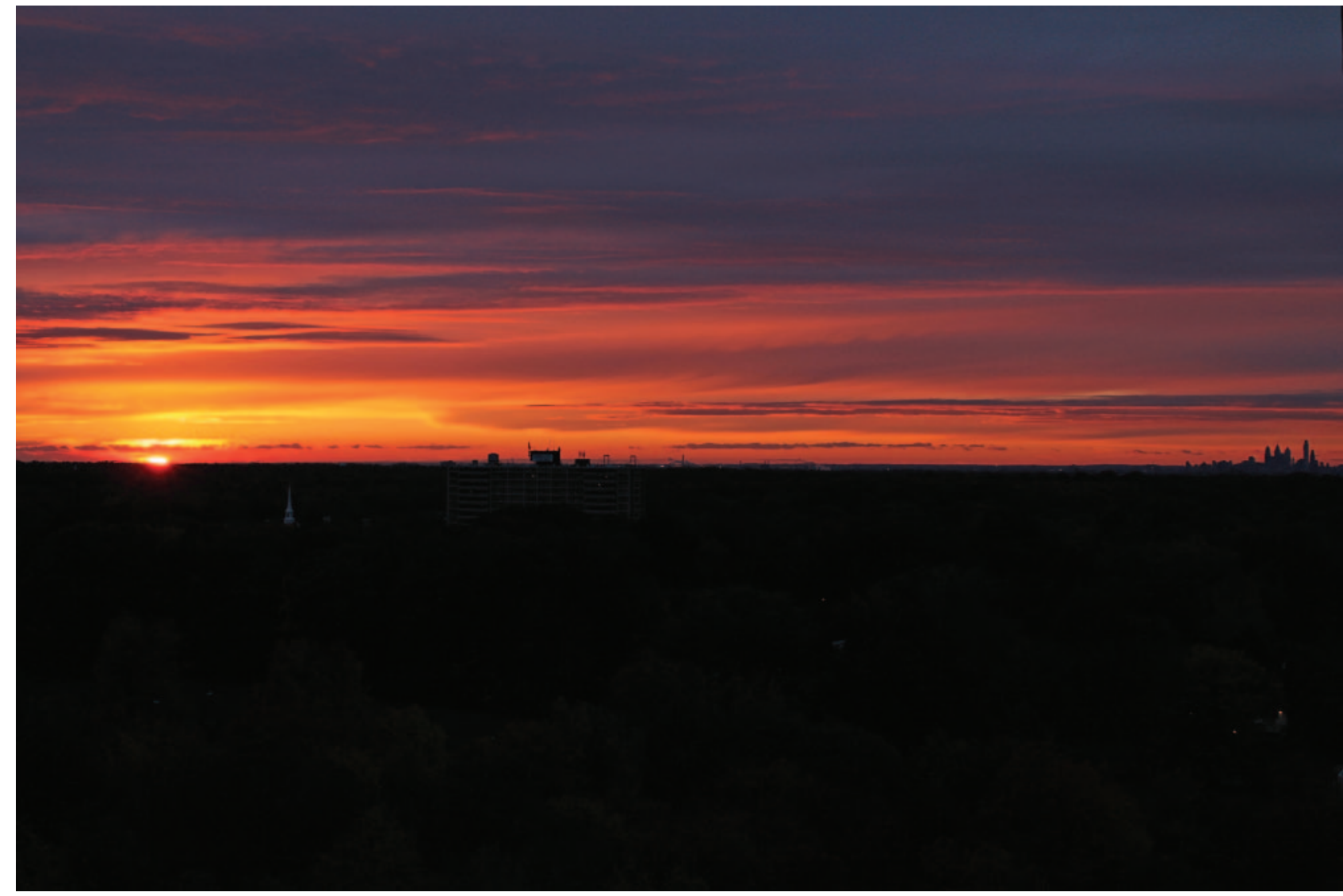

